\title{
Modeling of turbulent transport as a volume process
}

\author{
By MARK J. JENNINGS ${ }^{1}$ AND THOMAS MOREL ${ }^{2}$
}

\section{Introduction}

One of the issues in turbulence modeling is the representation of turbulent transport of Reynolds-averaged quantities, both mean values and turbulent statistics. The process of turbulent transport is one of dispersion due to the fluctuating turbulent motions, which span a wide range of spatial scales. In the current models of turbulence, some of the transport terms are calculated from transport equations for the terms themselves. Specifically, the turbulent transport of a quantity $\phi$ due to velocity- $\phi$ correlations may be calculated from higher-order equations obtained by manipulation of the $\phi$-equation and the momentum equation. However, each such equation introduces additional transport terms due to velocity and pressure correlations.

At any selected level of turbulence modeling, there remains a number of these correlations, and those are almost universally being modeled by gradient diffusion, analogous to the laminar diffusion, with an isotropic scalar diffusivity. More recently, Rogers, Mansour and Reynolds (1987) have proposed a model which employs tensorial diffusivity for heat transfer. The analogy of turbulent transport with laminar diffusion is only distant. This is in part because the seemingly random turbulent motions responsible for the transport can be relatively large compared to the scale of the entire flow region and thus they can communicate with adjacent flow regions. Also, in strained flows these motions have preferred directions and consequently their contributions are not isotropic.

The objective of this work has been to give consideration to an alternative class of methods for the representation of turbulent transport, which would incorporate the effects of (1) the surrounding finite volume, and (2) preferred lines of communication within the flow. These methods, if successful, should have the potential of removing the drawbacks of the current gradient diffusion models.

In a broader sense, all of the turbulence correlations appearing in the Reynoldsaveraged equations are the result of non-linear interactions of turbulent motions of various scales. Their modeling has traditionally followed the path of representation in terms of loçal variables, be it mean gradients or other turbulence correlations. This approach has been moderately successful, although much work remains. It is likely that modeling of terms other than turbulent transfer would also benefit from the consideration of the surrounding volume and of the preferred directions

1 Illinois Institute of Technology

2 Integral Technologies 
of strained turbulence. In fact, some work along these lines has already been done, e.g., by Miklavic and Wolfshtein (1987).

\section{Conceptual model of turbulent transport}

One concept for alternative description of turbulent transport is to represent it as net convection by fluctuating turbulent motions, which exchange small volumes of fluid between neighboring points. It is assumed that one needs to consider only points that are close enough to be able to influence each other directly. This is based on the premise that the spatial extent of turbulent transport is controlled by the extent over which the motions are coherent. That implies that the turbulent transport of any quantity is directly linked to the coherence and dynamics of the fluid motions. A further premise is that the influence of mean shear on the turbulent transport of a scalar quantity $\phi$ (which appears in the governing equations of velocity- $\phi$ correlations as a consequence of manipulation of the momentum equation) is accounted for indirectly, through the action of mean shear on the velocity fluctuation field, resulting in its distortion, anisotropy, and preferred lines of communications within it. The turbulent transport of the scalar can then be deduced from the interaction of the distorted turbulent velocity field with the spatial distribution (gradients) of the mean scalar $\Phi$.

Assuming that the above premises are valid, one may proceed to search for an appropriate formulation of the transport term, which would properly represent this physical picture. Consider two small volumes, one centered on a given point $\mathbf{x}$ and the other on some arbitrary point $x^{\prime}$ in the neighborhood of $\mathbf{x}$. These two volumes interact and exchange fluid carrying the scalar. Based on the discussion above, we propose to model the scalar transport in terms of the mean field $\Phi$ by

$$
T \phi_{i}(\mathbf{x}, t)=C \int\left[\Phi(\mathbf{x}, t)-\Phi\left(\mathbf{x}^{\prime}, t\right)\right] X D \frac{V}{L} \frac{r_{i}}{r} \frac{1}{4 \pi r^{2}} d^{3} \mathbf{r}
$$

where $\mathbf{r}=\mathbf{x}-\mathbf{x}^{\prime}, X$ is a dimensionless spatial function descriptive of the velocity fluctuation field, $V / L$ is the time scale of turbulent motions with $V$ being a velocity scale and $L$ an integral length scale, $D$ is a scalar function of $r / L$ discussed below, and $r_{i} / r$ is the direction cosine of the vector $\mathbf{r}$ in the $i$-direction. The factor $1 /\left(4 \pi r^{2}\right)$ is introduced to represent the decaying influence of a volume as the distance $r$ increases. The introduction of the direction cosine into the relationship implies that the contribution to transport between any two given points in the flow is directed along the vector connecting these points. The function $D$ is included to represent changes in the convected scalar during the transit time $\tau=r / L$ due to diffusion.

The general nature of equation (1) is such that the transport calculated using that expression can produce counter-gradient flux in certain types of non-homogeneous flows. Furthermore, this term is in general non-isotropic (depending on the function $X$ ) and thus is more general than commonly used scalar diffusivity models.

A key element in the model is the selection of the function $X$. Building on the premises of this approach, it seemed worthwhile to investigate the usefulness of basing this function on the two-point velocity correlation $Q_{r r}$ which correlates the 
velocity component along the vector $r$. Such a correlation can be shown to be related to the commonly used two-point correlation $Q_{i j}$

$$
Q_{i j}=\overline{u_{i}^{\prime}(\mathbf{x}, t) u_{j}^{\prime}\left(\mathbf{x}^{\prime}, t\right)}
$$

by a relation

$$
Q_{r r}=\frac{r_{i} r_{j} Q_{i j}}{r^{2}}
$$

The scalar $Q_{r r}$ is a function of the spatial position. In an isotropic flow this function has spherical contours centered on the correlating point and its value decays along any ray extending from the origin according to the longitudinal correlation function $f(r)$. In a shear flow this function is much more complex, as will be discussed below.

\section{Two-point velocity correlations}

\section{Homogeneous shear flow}

To test the ideas proposed above, advantage was taken of the available data produced through direct numerical simulations of turbulent flows at Stanford University and at NASA-Ames. One such flow was the homogeneous shear flow calculated by Rogers et al. (1986), specifically the flow C128U. Analysis was made of the twopoint correlations at dimensionless time $S t=12$. At that instant, the flow may be characterized by $S q^{2} / \epsilon=11, q^{4} / \epsilon \nu=800, \nu_{t} / \nu=13, P / \epsilon=1.7$, where $S$ is the mean shear rate $d U / d y, q^{2} / 2$ is the turbulent kinetic energy, $\epsilon$ is the turbulent dissipation rate, $P$ is the production rate, $\nu$ is the molecular kinematic viscosity, and $\nu_{t}$ is the effective turbulent kinematic viscosity. This is a fully-developed turbulent flow, albeit at fairly low Reynolds number as evidenced by the low value of $\nu_{t} / \nu$. The ratio of longitudinal length scale $\lambda_{1}$ to the computational grid spacing in the $x$-direction was about 5 , and the computational domain spanned 128 nodes in all three directions. The simulation included a cross-stream vertical gradient of a passive scalar with molecular Prandtl number of 0.7 .

Using the data base, two-point velocity-velocity and velocity-scalar correlations were obtained. The two-point $u_{1} u_{1}, u_{1} \phi, u_{2} u_{2}$, and $u_{2} \phi$ correlations are shown in Figures 1-4 for the $x-y$ and $y-z$ planes through the correlating point. Inspection of the contours shows a distinct similarity between the two types of correlations. The $u_{1} \phi$ correlation appears to be strongly influenced by the $u_{1} u_{1}$ correlation. The $u_{2} \phi$ contours are somewhat similar to the $u_{2} u_{2}$ contours, although there also appears to be some influence from the $u_{1} u_{2}$ correlation. This tends to support the view that two-point velocity correlations are relevant to scalar transport.

An interesting, and perhaps surprising observation, is that the contours of the $u_{2} u_{2}$ correlation have no inclination, while the $u_{1} u_{1}$ and $u_{3} u_{3}$ correlation contours are very strongly inclined in a direction that is consistent with the mean shear and with the orientation of the flow structures (hairpins) that develop in the flow. This surprising behavior of the vertical fluctuations has been previously observed in experiments, e.g., in a boundary layer by Kovasznay et al. (1970) and in homogeneous 

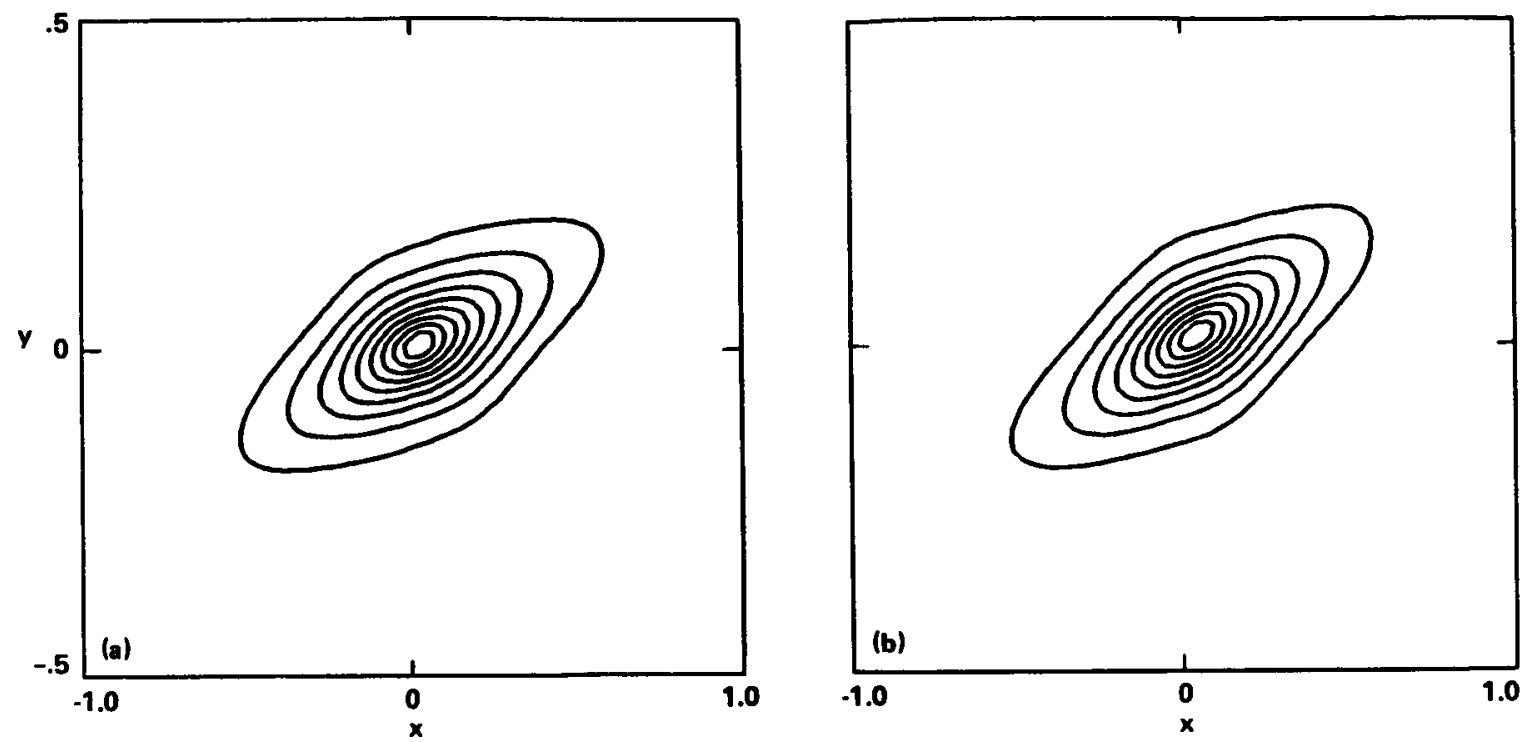

FIGURE 1. Comparison of velocity-velocity and velocity-scalar correlations in a homogeneous shear flow with a vertical mean temperature gradient. Note compressed $x$-scale. (a) $x-y$ plane, $u_{1} u_{1}$ and (b) $x-y$ plane, $u_{1} \phi$.
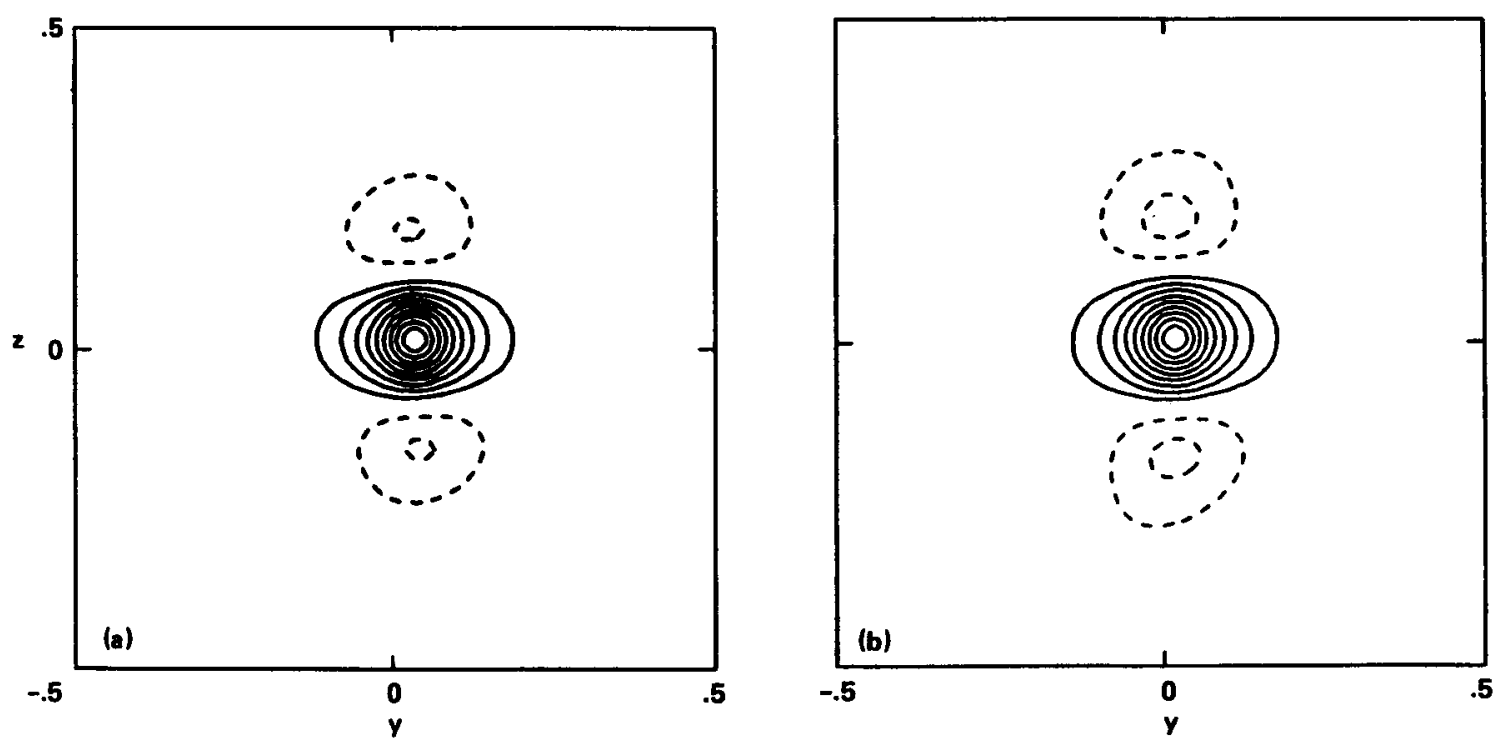

Figure 2. Comparison of velocity-velocity and velocity-scalar correlations in a homogeneous shear flow with a vertical mean temperature gradient. (a) $y-z$ plane, $u_{1} u_{1}$ and (b) $y-z$ plane, $u_{1} \phi$.

shear layer by Townsend (1970). The contours of the $u_{1} u_{2}$ correlation, being related to both $u_{1}$ and $u_{2}$, have an inclination which lies between the $u_{1} u_{1}$ and $u_{2} u_{2}$ inclinations.

The contracted correlation $Q_{r r}$, defined in equation (3), is a linear combination of 

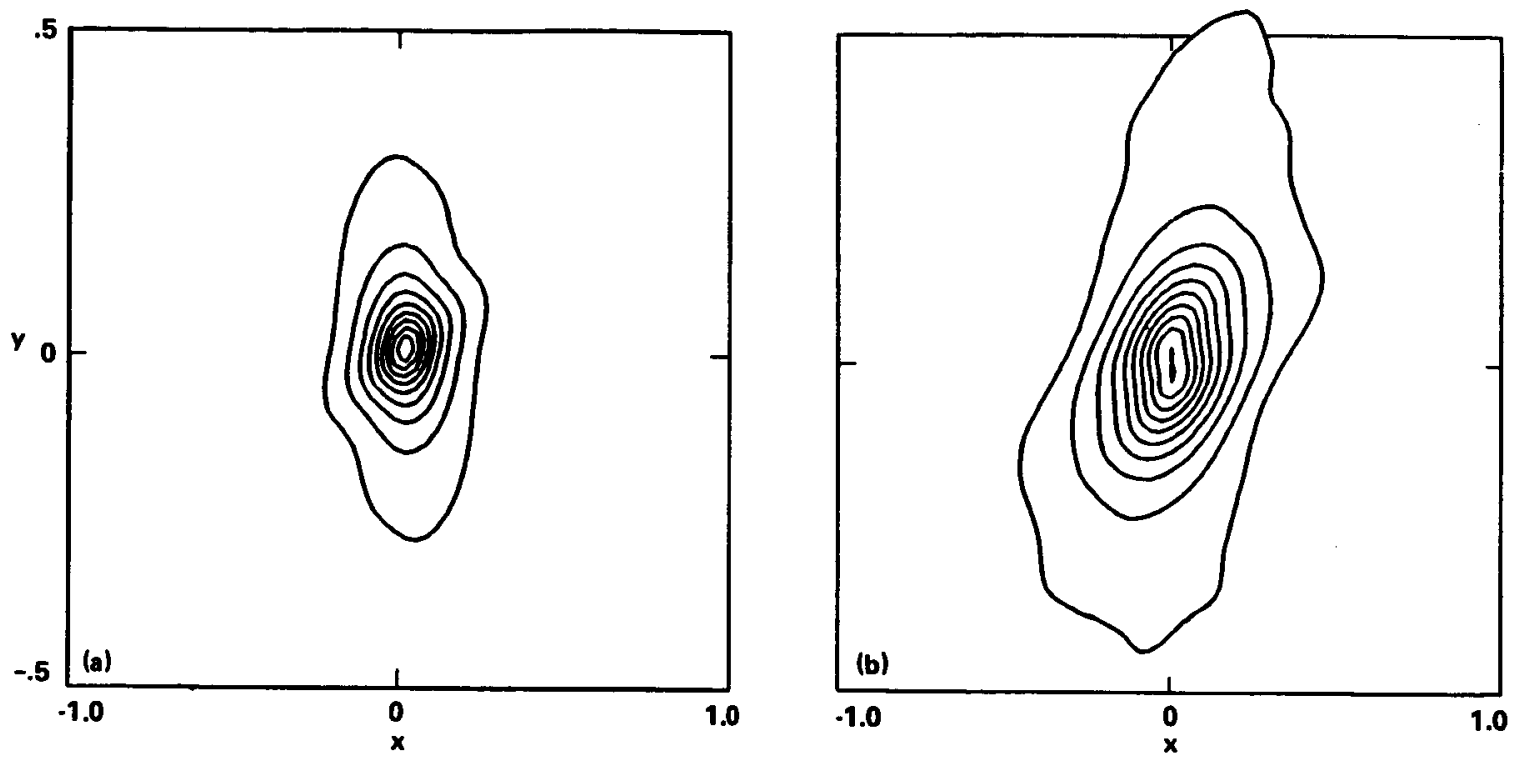

FIGURE 3. Comparison of velocity-velocity and velocity-scalar correlations in a homogeneous shear flow with a vertical mean temperature gradient. Note compressed $x$-scale. (a) $x-y$ plane, $u_{2} u_{2}$ and (b) $x-y$ plane, $u_{2} \phi$.
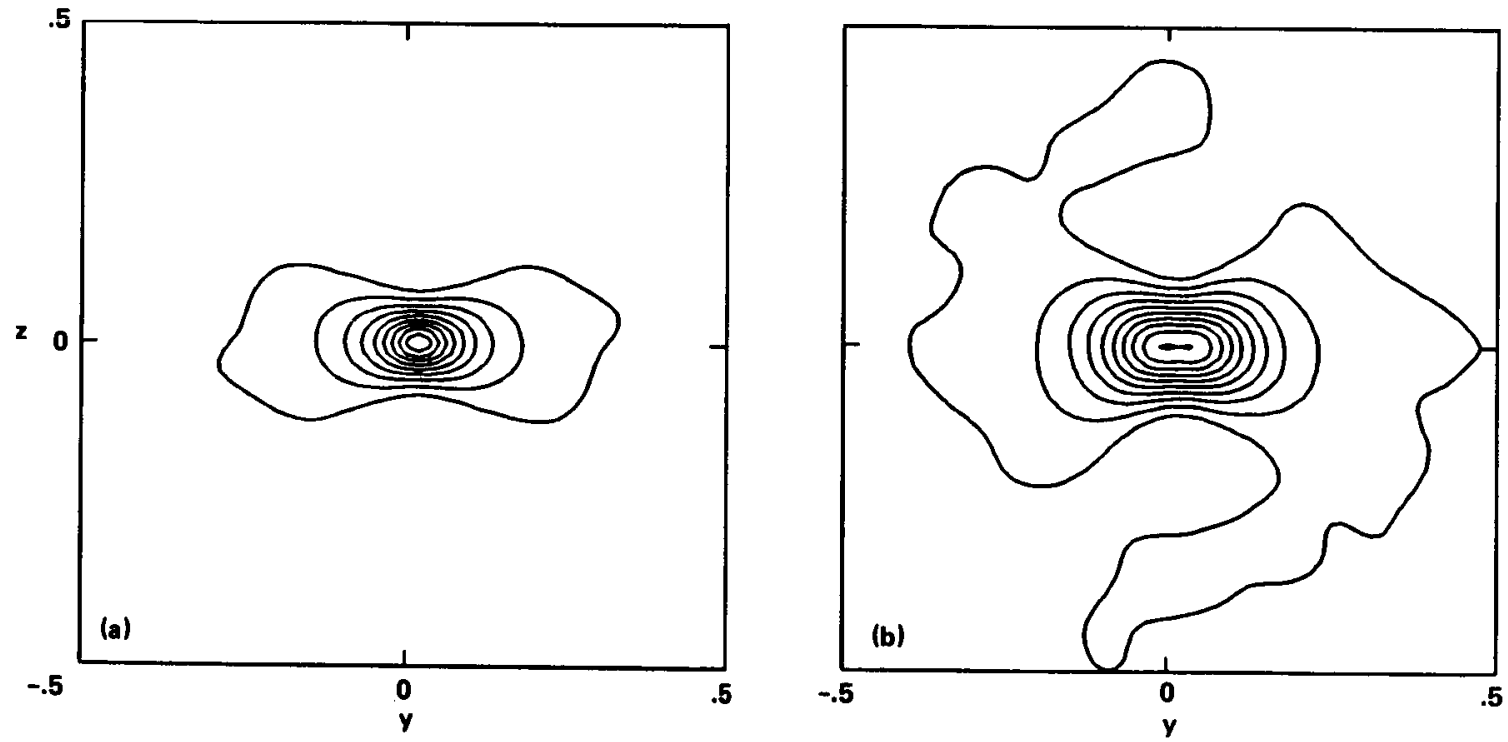

FIGURE 4. Comparison of velocity-velocity and velocity-scalar correlations in a homogeneous shear flow with a vertical mean temperature gradient. (a) $y$ - $z$ plane, $u_{2} u_{2}$ and (b) $y-z$ plane, $u_{2} \phi$.

the individual velocity correlations. Its contours are shown in Figure 5 for the three planes $x-y, y-z$, and $x-z$. It may be seen that this correlation is very different from the individual components $Q_{i j}$. Its values are mostly positive with only very weak 

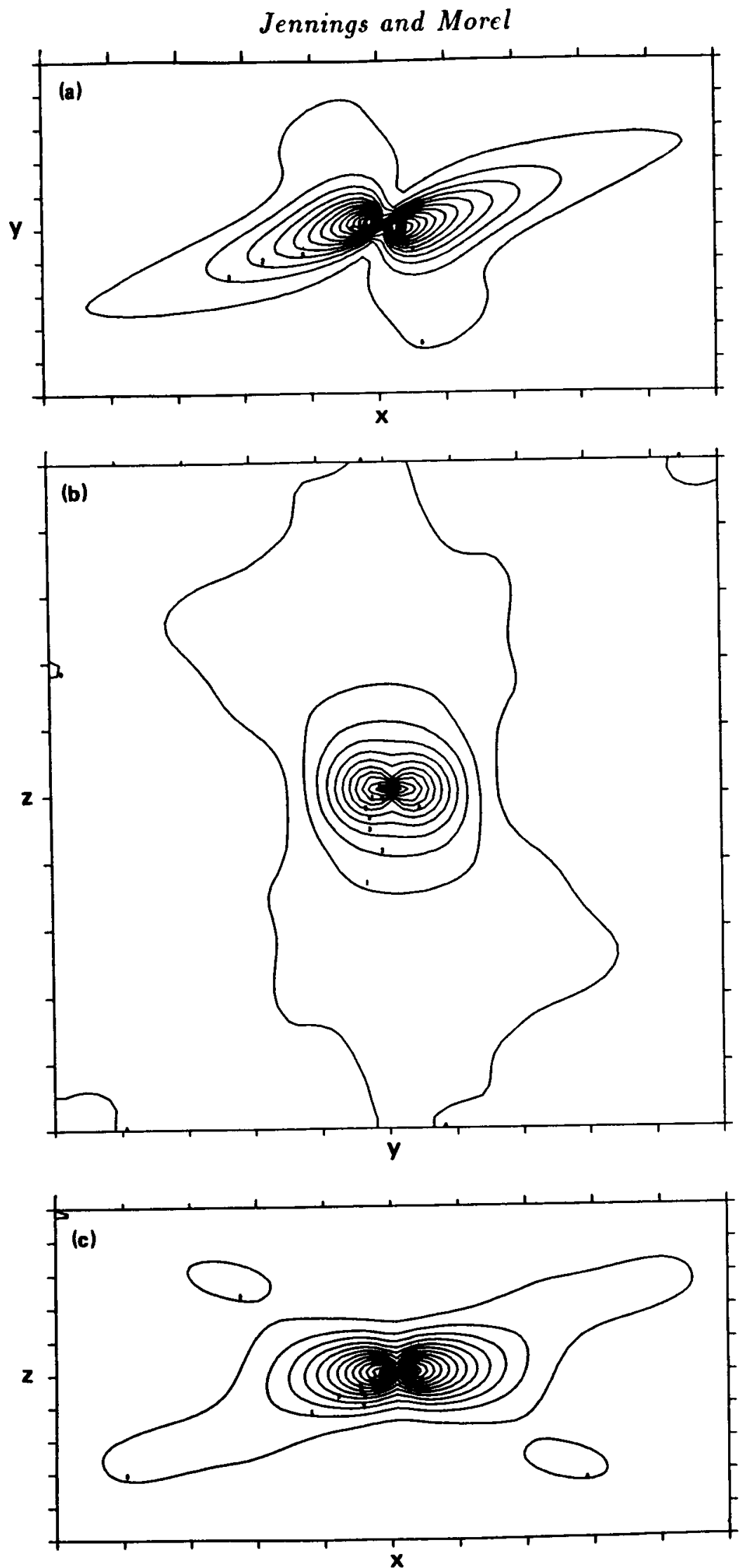

FIGURE 5. Contracted two-point correlation $Q_{r r}$. (a) $x-y$ plane; (b) $y-z$ plane and (c) $x-z$ plane. 
negative lobes. In the $x-y$ plane (Figure $5 \mathrm{a}$ ) this correlation has a very distinctive shape. This is partly caused by the fact that $Q_{r r}$ has a singular behavior at the origin, where its value depends on the direction from which this point is being approached. The butterfly-like shape seen in this plane is mainly produced by the contributions from the $Q_{12}$ and $Q_{21}$ correlations, and it is absent in the other two planes. In the spirit of the discussion above, this shape of the correlation volume may be interpreted as an indication of the preferred lines of communication set up in a strained turbulent field. It is seen that the preferred communication is along a direction close to the mean flow direction, while a very poor communication is set up along a direction inclined about 70 degrees from the $x$-direction. This anisotropy may be expected to have a major effect on turbulent transport and perhaps on other processes as well. The correlation volume reflects the effects of the dominant turbulent motions and should be consistent with their orientation and shape; in the case of the homogeneous shear flow these are hairpin-shaped, and their relationship to the correlation volume shape is not immediately obvious.

In the case of homogeneous shear flow equation (1) simplifies to

$$
T \phi_{i}=\frac{c}{4 \pi} \Phi_{, j} V \int Q_{r r} r_{i} r_{j} \frac{d \mathbf{r}}{r^{2}}
$$

or

$$
T \phi_{i}=-D_{i j} \Phi_{, j} .
$$

Evaluating the integral in equation (4) over the entire domain one obtains a tensorial diffusivity $D_{i j}$. The values calculated for this particular flow are given below:

$$
\begin{gathered}
\left(D_{i j} / D_{22}\right)_{\text {model }}=\left(\begin{array}{rrr}
1.80 & -0.23 & 0.05 \\
-0.23 & 1.00 & -0.04 \\
0.05 & -0.04 & 1.00
\end{array}\right) \\
\left(D_{i j} / D_{22}\right)_{\text {simulation }}=\left(\begin{array}{rrr}
6.5 & -2.4 & 0.0 \\
-1.1 & 1.0 & 0.0 \\
0.0 & 0.0 & 1.8
\end{array}\right) .
\end{gathered}
$$

The first of the two tensors is that calculated by the present model, the second one has the values deduced by Rogers et al. (1986) from the direct simulation. The model values are seen to exhibit correct trends, with high values of $D_{11}$ and with negative off-diagonal contributions $D_{12}$ and $D_{21}$. However, the magnitudes of the departures from isotropy are not predicted well. By contrast, scalar diffusivity models contain only diagonal terms that are equal in magnitude.

\section{Channel flow}

To complement the data presented above, a brief study was made of a two-point correlation in a channel flow simulation data of Kim, Moin and Moser (1987). The Reynolds number based on wall friction velocity $u_{\tau}$ and channel half-width $H$ was 180. The contours of the $u_{1} u_{1}, u_{2} u_{2}, u_{3} u_{3}$ and $u_{1} u_{2}$ two-point correlations at 
distances of one-quarter and one-half $H$ from the wall were computed. At the onequarter $H$ location, $u_{1} u_{1}$ and $u_{3} u_{3}$ contours are stretched and inclined towards the channel, wall. The $u_{2} u_{2}$ contours are seen to be affected by the presence of the wall, especially those at lower correlation levels which are farther away from the origin. This general behavior is also seen at the half $H$ point, except that the wall effects are diminished and some similarities with the homogeneous shear flow begin to emerge.

\section{Summary}

An alternative type of modeling has been proposed for the turbulent transport terms in Reynolds-averaged equations. During the Summer Program, one particular implementation of the model has been considered, based on the two-point velocity correlations. The model was found to reproduce the trends but not the magnitude of the non-isotropic behavior of the turbulent transport. Some interesting insights have been developed concerning the shape of the contracted two-point correlation volume. This volume is strongly deformed by mean shear from the spherical shape found in unstrained flows. Of particular interest is the finding that the shape is sharply waisted, indicating preferential lines of communication, which should have a direct effect on turbulent transfer and on other processes.

\section{Acknowledgements}

We wish to acknowledge the able and generous support provided to us during the Summer Program at CTR by all involved. Special recognition is due to Scott Abrahamson, Sanjiva Lele, Bob Moser, and Mike Rogers.

\section{REFERENCES}

KIM, J., MoIn, P.. \& Moser, R. D. 1987 Turbulence statistics in fully developed channel flow at low Reynolds number. J. Fluid Mech. 177, 133-166.

Kovasznay, L. S. G., Kibens, V. \& Blackwelder, R. F. 1970 Large-scale motion in the intermittent region of a turbulent boundary layer. J. Fluid Mech. 41, 283-325.

Miklavic, S. J. \& Wolfshtein, M. 1987 A quasi-isotropic model of the twopoint temperature-velocity correlation and its application to the modeling of the turbulent heat flux, to be published.

Rogers, M. M., Mansour, P. \& Reynolds, W. C. 1987 An algebraic model for the turbulent flux of a passive scalar, to be published.

Rogers, M. M., Moin, P. \& Reynolds, W. C. 1986 The structure and modeling of the hydrodynamic and passive scalar fields in homogeneous turbulent shear flow. Dept. Mech. Eng. Report No. TF-25. Stanford University, Stanford, California.

Townsend, A. A. 1970 Entrainment and structure of turbulent flow. J. Fluid Mech. 41, 13-46. 